\title{
Hoping to indirectly detect Dark Matter with cosmic rays
}

\author{
Marco Cirelli \\ CERN Theory Division, PH-TH, Case C01600, CH-1211, Geneva 23, Switzerland \\ and \\ Institut de Physique Théorique, CNRS URA 2306 \& CEA, F-91191 Gif-sur-Yvette, France
}

\begin{abstract}
Dark Matter constitutes more that $80 \%$ of the total amount of matter in the Universe, yet almost nothing is known about its nature. A powerful investigation technique is that of searching for the products of annihilations of Dark Matter particles in the galactic halo, on top of the ordinary cosmic rays. Recent data from the PAMELA and FERMI satellites and a number of balloon experiment have reported unexpected excesses in the measured fluxes of cosmic rays. Are these the first direct evidences for Dark Matter? If yes, which DM models and candidates can explain these anomalies (in terms of annihilations) and what do they imply for future searches? What are the constraints from gamma rays measurements and cosmology?
\end{abstract}

[Report number: Saclay T-10/098, CERN-PH-TH/2010-183]

Keywords: Dark Matter, Indirect Detection, Cosmic Rays

PACS: $95.35 .+\mathrm{d}, 96.50 . \mathrm{S}-$, 12.60.-i

\section{INTRODUCTION}

While compelling evidence for the existence of Dark Matter (DM) now comes from a number of astrophysical and cosmological probes, no explicit detection has been confirmed yet. The indirect detection strategy relies on the possibility of seeing signals of the presence of DM in terms of the final products $\left(e^{ \pm}, p, d, \gamma, v \ldots\right)$ of DM annihilations in the galactic halo, on top of the ordinary cosmic rays. The recent positive results from a number of indirect detection experiments have suggested the possibility that indeed such a signal has been seen. In particular, the signals point to an excess of electrons and positrons.

- Data from the PAMELA satellite show a steep increase in the energy spectrum of the positron fraction $e^{+} /\left(e^{+}+e^{-}\right)$above $10 \mathrm{GeV}$ up to $100 \mathrm{GeV}$, compatibly with previous less certain hints from HEAT and AMS-01.

- Data from PAMELA also show no excess in the $\bar{p} / p$ energy spectrum compared with the predicted background.

- The balloon experiments ATIC-2 and PPB-BETS report the presence of a peak in the $e^{+}+e^{-}$energy spectrum at around 500-800 GeV.

- This sharp feature is however questioned by the results of the FERMI satellite: while an excess with respect to the expected background is confirmed, the $e^{+}+e^{-}$ spectrum is found to be instead reproduced by a simple power law.

- The HESS telescope also reports the measurement of the $e^{+}+e^{-}$energy spectrum above energies of $600 \mathrm{GeV}$, showing a power law spectrum in agreement with the

CP1304, Carpathian Summer School of Physics 2010 edited by L. Trache, S. Stoica, and A. Smirnov

(C) 2010 American Institute of Physics 978-0-7354-0859-3/10/\$30.00 
one from FERMI and eventually a steepening at energies of a few $\mathrm{TeV}$.

In this presentation I will address the following issues (the discussion in based on $[1,2$, $3,4,5]$, where all references are given):

- Which characteristics must a DM candidate have in order to fit the above data?

- What are the constraints from other observations (e.g. diffuse galactic $\gamma$ rays)?

- Are there constraints from cosmological observations?

- Which conclusions can then be drawn on the DM interpretation of the data?

\section{POSITRONS, ELECTRONS AND ANTIPROTONS: WHICH DARK MATTER CAN FIT THE DATA?}

As a first example, the upper row of Fig. 1 shows the spectra of the positron fraction (first column), of the sum of electrons and positrons (second column) and of the antiprotons (third column) from a DM particle with $150 \mathrm{GeV}$ mass and annihilating into $W^{+} W^{-}$. As apparent, the candidate can fit well the positron data, but produces too large a flux of antiprotons: such a DM is excluded by data with pretty high confidence, unless a serious misunderstanding of the $\bar{p}$ background is invoked. Let us instead consider (second row of Fig.1) a candidate with a (very large) $10 \mathrm{TeV}$ mass, again annihilating into $W^{+} W^{-}$. The positron data points are well fitted (by the low energy tail of the spectrum, in this case) and the antiproton bounds are not exceded, thanks to the fact that an excess would show only at larger energies. However the features reported in the $e^{+}+e^{-}$spectrum are not reproduced. In the third row we consider a $1 \mathrm{TeV}$ candidate with annihilations into $\mu^{+} \mu^{-}$: it fits the PAMELA data in $e^{+}, \bar{p}$ (which are not produced by the purely leptonic channel) and it reproduces the peak in the $e^{+}+e^{-}$spectrum hinted to by ATIC. As a final exemple, we consider a sligthly heavier $(3 \mathrm{TeV})$ candidate with annihilations into $\tau^{+} \tau^{-}$(fourth row of Fig. 1): it fits the PAMELA, FERMI and HESS datasets.

We now proceed to presenting the results of the fits in a more systematic way. In performing such fits, we smoothly scan over the charged cosmic ray propagation configurations and DM halo models, within the boundaries described in [1]. Moreover, we assume that the $e^{+}, e^{-}, \bar{p}$ background spectra can be freely renormalized, and have independent \pm 0.05 errors in their energy slope. This mimics the main uncertainties in astrophysical backgrounds. We will show plots of the $\chi^{2}$ as a function of the DM mass: an interval at $n$ standard deviations corresponds (in Gaussian approximation) to $\chi^{2}<\chi_{\min }^{2}+n^{2}$, irrespectively of the number of data points.

First, let us consider the fit to PAMELA positron data only (16 data points). We see in the upper left panel of fig. 2 that DM annihilations into $e, \mu, \tau, W$ can reasonably well reproduce the data for any $\mathrm{DM}$ mass, while annihilations into $Z, t, q, b, h$ give a good fit for $\mathrm{DM}$ heavier than about $1 \mathrm{TeV}$. It is perhaps interesting to note that, contrary to what commonly thought, the spectrum from $W^{+} W^{-}$annihilations is not too flat to give a good fit of the quite steep PAMELA rise.

Next, let us add the PAMELA $\bar{p} / p$ data (17 data points). Since no excess seems present in the $\bar{p} / p$ ratio, annihilation into leptons are not constrained as they do not produce antiprotons. On the contrary, all other annihilations into quarks, vector and Higgs 
DM with $M=150 \mathrm{GeV}$ that annihilates into $W^{+} W^{-}$
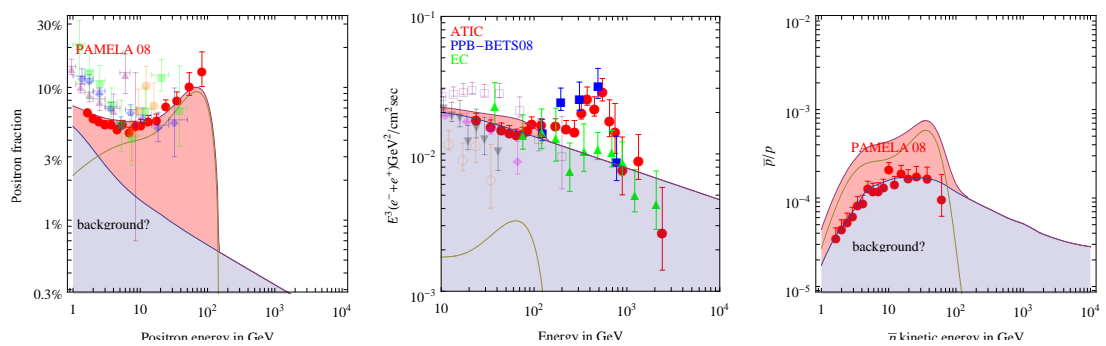

DM with $M=10 \mathrm{TeV}$ that annihilates into $W^{+} W$
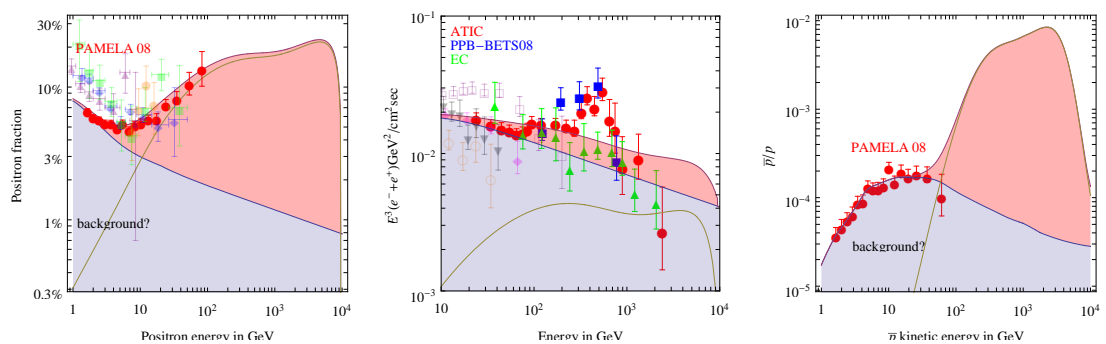

DM with $M=1 \mathrm{TeV}$ that annihilates into $\mu^{+} \mu^{-}$
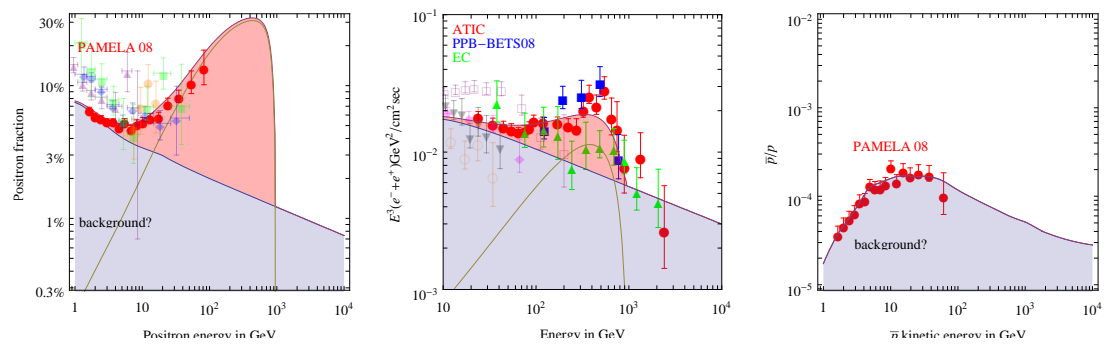

DM with $M=3 \mathrm{TeV}$ that annihilates into $\tau^{+} \tau^{-}$
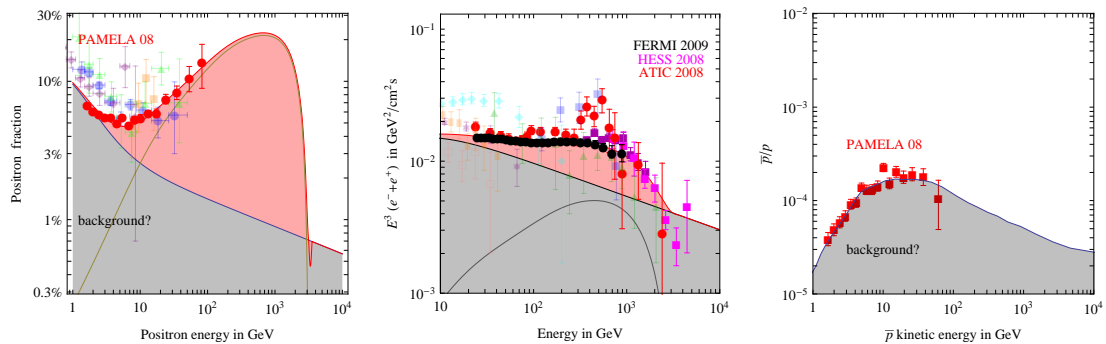

FIGURE 1. Four examples of fits of $e^{+}$(left), $e^{+}+e^{-}$(center), $\bar{p}$ (right) data, for a DM particle with mass $M=150 \mathrm{GeV}$ annihilating into $W^{+} W^{-}$(upper row, excluded by $\bar{p}$ ), $M=10 \mathrm{TeV}$ into $W^{+} W^{-}$ (second row, disfavored by the $e^{+}+e^{-}$data), $M=1 \mathrm{TeV}$ into $\mu^{+} \mu^{-}$(third row, in agreement with ATIC data) and $M=3 \mathrm{TeV}$ into $\tau^{+} \tau^{-}$(lower row, favored by FERMI and HESS). Galactic DM profiles and propagation models are varied to provide the best fit. 

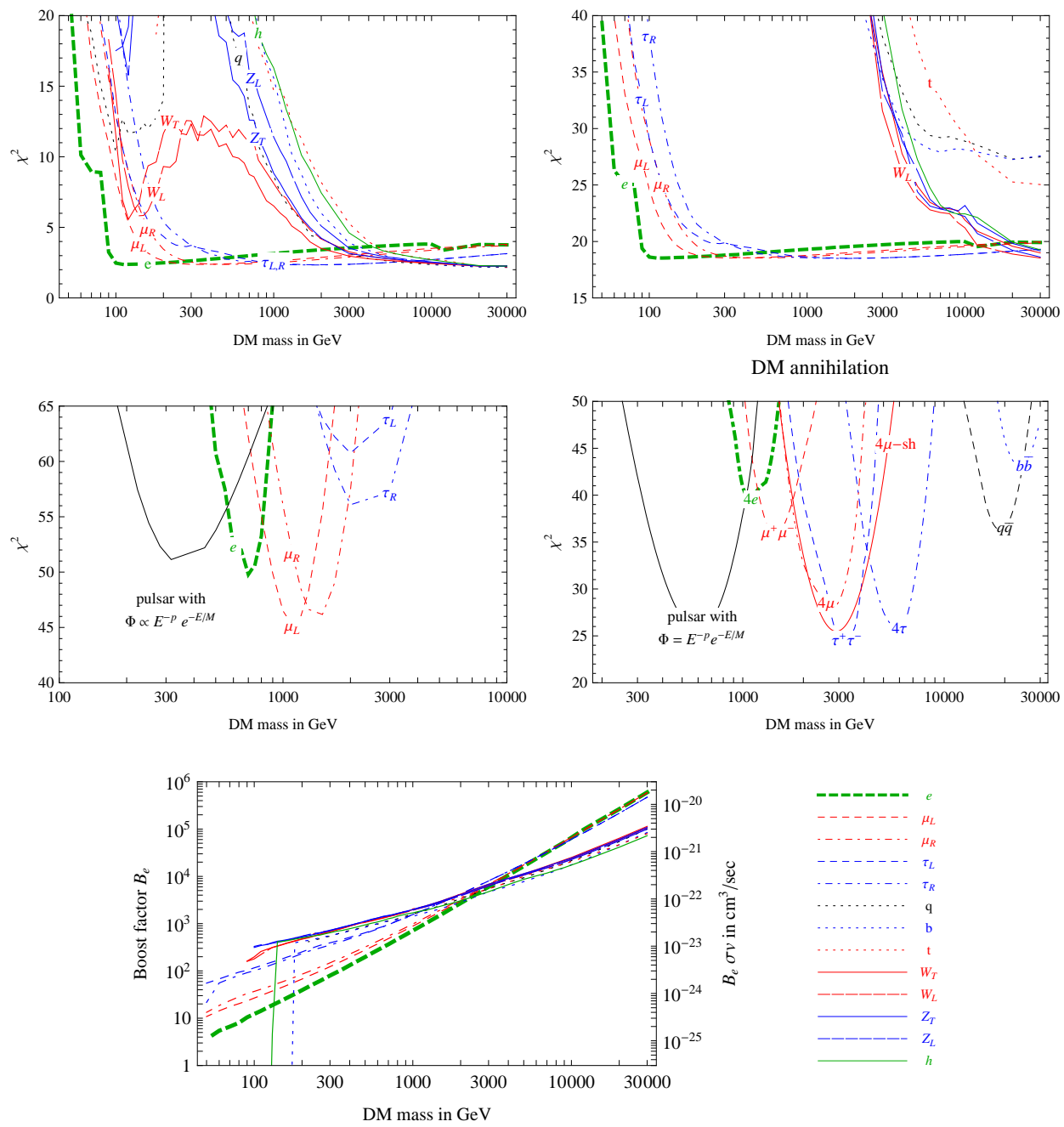

FIGURE 2. Global fits of different DM annihilation channels to the data. The labels on each curve indicate the primary annihilation channel. Upper left: fit to PAMELA $e^{+}$fraction data only. Upper right: with the inclusion of PAMELA antiproton data. Lower left: with the inclusion of balloon $e^{+}+e^{-}$data. Lower right: replacing balloon data with FERMI and HESS data (figure from P.Meade, M.Papucci, A.Strumia, T.Volansky, arXiv: 0905.0480; the four-lepton lines refer to exotic channels which are not discussed here). Bottom: values of $B_{e} \cdot \sigma v$ (right axis) and of the boost factor $B_{e}$ (left axis, for $\sigma v=$ $310^{-26} \mathrm{~cm}^{3} / \mathrm{sec}$ ) needed to fit the data.

bosons are significantly constrained, and allowed only if the DM particle is heavier than almost $10 \mathrm{TeV}$ (see the upper left panel of fig. 2). Only in such a case the proton excess lays at energies above those explored currently by PAMELA, while the low energy pro- 
ton spectrum is consistent with the background (see Fig. 1 for illustration). The bound dominantly comes from high energy data points where the solar modulation is negligible. The implications of the complementarity of PAMELA $e^{+} /\left(e^{+}+e^{-}\right)$and $\bar{p} / p$ data on constraining new physics are therefore evident.

We add now to the fit the balloon (ATIC-2, PPB-BETS and EC) data (37 points in total). Because the balloon data shows a sharp cut-off in the excess just below $1 \mathrm{TeV}$, the DM mass should be close to $1 \mathrm{TeV}$, and all other but leptonic DM annihilation channels are strongly disfavored or excluded. This is shown in the lower left panel of Fig. 2. More precisely, DM annihilations into $\mu$ seem to give the optimal energy spectrum and the best fit (see e.g. the example discussed above in Fig.1).

Finally, replacing the balloon data by the FERMI and HESS data (lower right panel of Fig. 2) modify slightly the best fit in favor of a candidate with a somewhat higher mass $(\sim 3 \mathrm{TeV})$ and a channel producing somewhat smoother leptonic spectra such as $\tau \tau$.

The lowermost panel of Fig. 2 illustrates the last important point: the values of the annihilation cross section which are required in order to fit the data (for a given mass and given primary annihilation channel). Values of the order of $10^{-23} \mathrm{~cm}^{3} / \mathrm{sec}$ or more (for the masses under consideration) are needed.

\section{CONSTRAINTS FROM PROMPT GAMMA RAYS, ICS GAMMA RAYS AND RADIO OBSERVATIONS}

Given these tantalizing but surprising hints of Dark Matter annihilations in the charged particle signals, it is now crucial to consider the constraints on this interpretation that come from the photon fluxes that necessarily accompany them. These photon fluxes are produced:

i) Directly as a product of the DM annihilations themselves (mainly from the bremsstrahlung of charged particles and the fragmentation of hadrons, e.g. $\pi^{0}$, produced in the annihilations), at energies comparable to the DM mass $M$, i.e. in the $\gamma$-ray energy range of tens of GeV to multi-TeV.

ii) By the Inverse Compton Scattering (ICS) of the energetic electrons and positrons, produced in the DM annihilation, onto the low energy photons of the CMB, the galactic star-light and infrared-light, which are thus upscattered up to energies again comparable to the DM mass.

iii) At much lower energies, e.g. radio frequency, by the synchrotron radiation emitted in the galactic magnetic field by the $e^{ \pm}$produced by DM annihilations.

The best targets to search for these annihilation signals are regions with high DM densities, such as the Milky Way Galactic Center (GC), the Milky Way Galactic Ridge (GR) and the Sagittarius Dwarf spheroidal satellite galaxy (Sgr dSph). In the case of the ICS signal, however, an intense flux is expected also from the regions of the galactic halo outside of the messy GC region, a feature which makes this a very interesting and more robust signature. The predicted photon fluxes can then be compared with observational data, in order to rule out combinations of astrophysical and particle physics parameters that violate observational constraints. The results of this are shown in fig. 3, for the case 
$\mathrm{DM} \mathrm{DM} \rightarrow e^{+} e^{-}$, Einasto profile

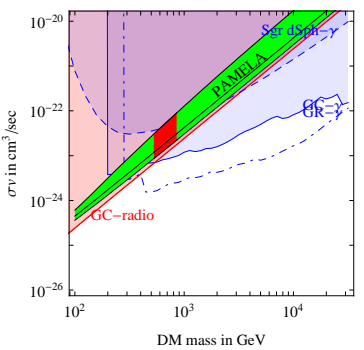

DM DM $\rightarrow$ ee, Einasto profile

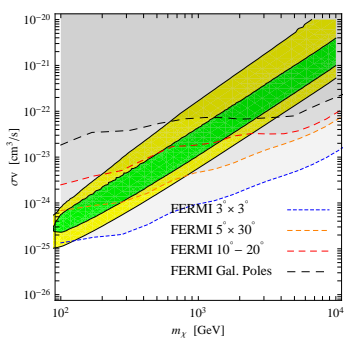

DM DM $\rightarrow$ ee, Einasto profile

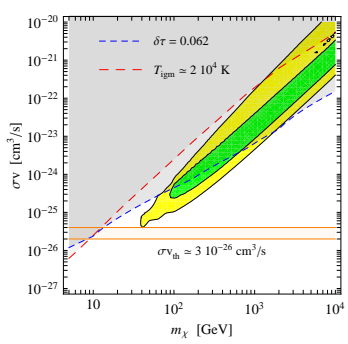

$\mathrm{DM} \mathrm{DM} \rightarrow \mu^{+} \mu^{-}$, Einasto profile

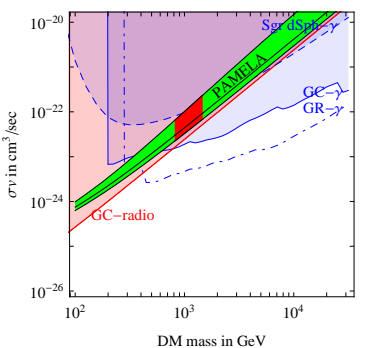

DM DM $\rightarrow \mu \mu$, Einasto profile

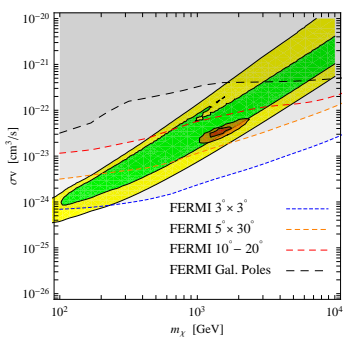

DM DM $\rightarrow \mu \mu$, Einasto profile

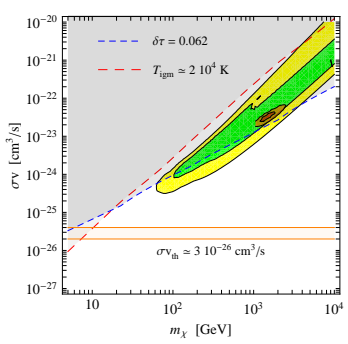

DM DM $\rightarrow \tau^{+} \tau^{-}$, Einasto profile

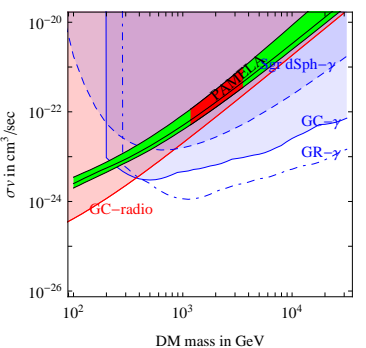

$\mathrm{DM}$ DM $\rightarrow \tau \tau$, Einasto profile

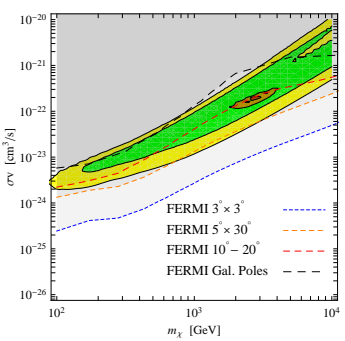

$\mathrm{DM} \mathrm{DM} \rightarrow \tau \tau$, Einasto profile

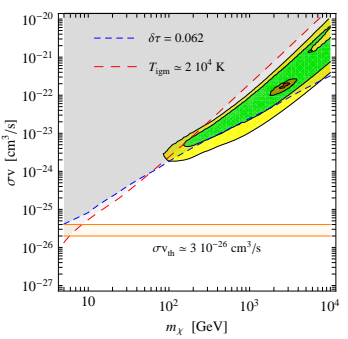

FIGURE 3. Comparison of the regions favored by PAMELA (green bands) + ATIC (red or yellow vertical regions within the bands) or + FERMI+HESS (orange blobs) with the bounds from gamma rays and cosmology. First row: constraints from HESS observations of the Galatic Center (blue continuous line), Galactic Ridge (blue dot-dashed), and SgrDwarf (blue dashed) and of observations of the GC at radio-frequencies by Davies et al. (red lines). Second row: constraints from EGRET and (preliminary) FERMI data of large windows in the galactic halo. Third row: constraints from the optical depth of the Universe and the temperature of the Intergalactic Medium. We considered DM annihilations into $e^{+} e^{-}$ (left), $\mu^{+} \mu^{-}$(middle), $\tau^{+} \tau^{-}$(right) and an Einasto DM density profile for the Milky Way and a density profile for Sgr dSph characterized by a large core. We assume unit boost and Sommerfeld factors.

of leptonic annihilations and choosing a benchmark Einasto profile. The first row shows the bounds imposed by prompt gamma rays and radio waves (shaded areas are excluded). The second row shows the constraints from ICS gamma rays using different observations from FERMI. It is apparent that the regions identified by PAMELA (and ATIC/FERMI) are excluded by these constraints. It is possible to relax them by assuming a less steep 
DM profile, such as isothermal (less DM concentration in the GC region leads to less gamma ray production), which is however disfavored by numerical simulations. For a full discussion, see Ref. [2], [3] and [5].

\section{CONSTRAINTS FROM THE REIONIZATION HISTORY}

The flux of energy injected by DM annihilation (all the way from the recombination epoch down to today through the formation history of DM halos) results in ionization and heating of the intergalactic medium. One way to constrain DM annihilation properties is therefore to look at the total optical depth of the Universe $\tau$, which results from the free electrons produced by the ionization. $\tau$ is measured by WMAP to be $\tau=0.084 \pm 0.016$, of which about 0.038 due to the low-redshift reionization $(z<6)$ produced by stars. A DM-induced optical depth larger than 0.062 (the $1 \sigma$ upper bound of the above) is therefore excluded by these arguments. Another way of assessing the impact of DM annihilations is to follow the evolution of the temperature of the intergalactic medium: temperatures higher that about $210^{4} \mathrm{~K}$ at low redshift contradict observations.

The third line of Fig. 3 shows such excluded regions on the usual plane 'DM mass' vs 'Annihilation cross section', for the same benchmark cases of leptonic channels and an Einasto profile. Again one finds that large portions of the regions that allow to fit the PAMELA and FERMI+HESS CR excesses in term of DM annihilations are ruled out by the optical depth bound. For instance, the entire PAMELA and FERMI+HESS region for the $\tau^{+} \tau^{-}$or $\mu^{+} \mu^{-}$case around $m_{\chi}=2$ or $3 \mathrm{TeV}$ is excluded. For a full discussion, see Ref. [4].

\section{GENERIC IMPLICATIONS ON DM MODEL BUILDING}

The possible signals (and constraints) discussed above identify a DM particle with peculiar features: a $\mathrm{TeV}$ or multi-TeV mass, very suppressed hadron production and a huge annihilation cross section, of the order of $10^{-23} \mathrm{~cm}^{3} / \mathrm{sec}$, much larger than the typical one required by DM thermal production in cosmology $\left(\sim 3 \cdot 10^{-26} \mathrm{~cm}^{3} / \mathrm{sec}\right)$. These peculiarities have stimulated many directions of model building activity.

The huge discrepancy in the annihilation rate can be justified in specific models in terms of some enhancement mechanism which is effective today but not in the Early Universe $^{1}$ : (i) The presence, in today's galactic halo, of DM overdensities that boost the annihilation rate (proportional to the squared density of DM particles): these however have been proven to be able to provide an $\mathscr{O}(10)$ boost at most. (ii) A Breit-Wigner resonance in the annihilation cross section: if the resonance mass is just below twice the DM mass, the annihilation cross section becomes sensitive to the details of the velocity distribution of the DM particles; since (on average) DM particles are slower today than in the Early Universe, many more of them meet the conditions of resonant

\footnotetext{
1 This assumes that one wants to preserve the production process of DM as a thermal relic. It is also possible, however, to postulate different, non-thermal production mechanisms, in which case the annihilation cross section can have been very large also in the Early Universe.
} 
annihilation and therefore the rate is enhanced, provided that the relevant parameters are appropriately fine tuned. (iii) The existence of a 'Sommerfeld enhancement': a nonperturbative effect which modifies the annihilation cross section in the regime of small relative velocity of the annihilating particles and in presence of an effectively long-range force between them. Indeed this well known quantum mechanical effect, first discussed by Sommerfeld in the context of positronium $e^{+} e^{-}$annihilations under the effect of resummed $\gamma$ exchanges, can occur in DM annihilations if the two annihilating particles exchange an interaction mediated by a force carrier of mass $m_{V}$ and with a coupling constant $g \approx \sqrt{4 \pi \alpha}$ such that $\alpha M_{\mathrm{DM}} / m_{V} \gtrsim 1$. For very heavy DM particles $(\gtrsim 10 \mathrm{TeV})$, the exchange of SM weak bosons can mediate the effect, in which case $\alpha$ is just the one of weak interactions and $m_{V} \approx m_{W^{ \pm} . Z} \approx 100 \mathrm{GeV}$. If a new force exist, however, mediated by a particle with mass $m_{V} \approx 1 \mathrm{GeV}$ and gauge-like coupling strength with DM particles only, even DM particles of mass $\lesssim 1 \mathrm{TeV}$ would enjoy the Sommerfeld enhancement. As an added bonus, DM annihilations into on-shell $V$ would not produce $\bar{p}$, their mass being higher, and guarantee in this way the leptophilic nature. This line of model building (1 TeV DM particle with a new 'dark force' mediated by a sub-GeV particle) has thriven in the recent past (see e.g. [1, 6, 7], among many incarnations).

The possibility has also been considered that the signals observed by PAMELA and FERMI+HESS be due to DM particle decays (see e.g. [8, 9], among many): if the decay rate is tuned to $\approx 10^{26}$ seconds (a figure possibly motivated by some high enegy physics scale suppressed operators) and if the production of hadrons is adequately suppressed by some a priori unrelated mechanism, the features needed to fit the data are obtained. A collateral advantage is that gamma ray constraints are less severe in these scenarios, as they are proportional to the first power of the DM density (and not the second, like for annihilations). For a full discussion on constraints, see however Ref. [5].

\section{CONCLUSIONS}

En lieu of conclusions, let us try to answer the questions raised in the Introduction.

- Which characteristics must a DM candidate have in order to fit the above data?

a) on the basis of the $e^{+}$and $\bar{p}$ data from PAMELA, the Dark Matter can be:

a1) a particle that dominantly annihilates into leptons, with no strong preference for the mass, if above a few hundred GeV;

a2) a particle that annihilates into $W, Z$ or $h$ and that has a mass $\gtrsim 10 \mathrm{TeV}$.

b) adding the peak from ATIC, a clear indication for the mass emerges: DM has to be a particle with mass $\sim 1 \mathrm{TeV}$ that dominantly annihilates into leptons.

c) replacing the ATIC peak with the FERMI smoother spectrum and the indication for a cutoff at a few TeV from HESS shifts somewhat the best fit, but not the main features: DM has to be a particle with mass $\sim 3 \mathrm{TeV}$ that dominantly annihilates into leptons ( $\tau$ is best).

Models with $M \ll 1 \mathrm{TeV}$ appear anyway to be already disfavored. For what concerns the magnitude of the annihilation cross section, the large flux above the background in the PAMELA data indicates a very large $\sigma v$, of the order of $10^{-23} \mathrm{~cm}^{3} / \mathrm{sec}$ or more (see lower right panel of Fig. 2). 
- What are the constraints from other observations (diffuse galactic $\gamma$ rays)?

Constraints are imposed by high energy gamma rays (generated directly from the DM annihilation process or by the ICS upscattering of the CMB and starlight photons) from the galactic center region and from satellite galaxies and by synchrotron radiation (generated by $e^{ \pm}$in the galactic center's magnetic field). The results show that the regions of the parameter space that allow to fit the PAMELA (and ATIC or FERMI+HESS) data are disfavored by about one order of magnitude if a benchmark Einasto (or NFW) profile is assumed. Choosing a smoother profile and/or assuming that a part of the cross section is due to an astrophysical boost factor that would not be present in dwarf galaxies and the Galactic Center due to tidal disruption re-allows part of the space. ICS constraints are however more robust and more difficult to circumvent with these arguments. It is fair to say that a tension is present between the charged CR signals and the gamma ray constraints.

- Are there constraints from cosmological observations?

Yes, robust constraints are imposed by the reionization history of the Universe: DM annihilation that allow to explain PAMELA+FERMI+HESS tend to produce too many free electrons that make the Universe more opaque than what is observed.

- Which conclusions can be drawn on the Dark Matter interpretation of the data? As apparent, the data point to a Dark Matter particle that (1) features really 'unexpected' properties and (2) has anyway disturbing 'internal' tensions (with $\gamma$ ray constraints and cosmology). So, either the DM interpretation is not the right one, i.e. an astrophysical source will turn out to be responsible for the excesses. Or we are on the verge of a big change of paradigm in the field of Dark Matter modelling.

\section{ACKNOWLEDGMENTS}

I thank my collaborators Alessandro Strumia, Gianfranco Bertone, Carolin Braüninger, Mario Kadastik, Fabio Iocco, Paolo Panci, Martti Raidal, Pasquale D. Serpico and Marco Taoso for the work presented here. I thank the EU Marie Curie Research \& Training network "UniverseNet" (MRTN-CT-2006-035863) for support. I especially thank the organizers of the 'Carpathian Summer School of Physics 2010' for the very enjoyable atmosphere.

\section{REFERENCES}

1. M. Cirelli, M. Kadastik, M. Raidal and A. Strumia, Nucl. Phys. B 813 (2009) 1 [0809.2409 [hep-ph]].

2. G. Bertone, M. Cirelli, A. Strumia and M. Taoso, JCAP 0903 (2009) 009 [arXiv:0811.3744 [astro-ph]].

3. M. Cirelli and P. Panci, Nucl. Phys. B 821 (2009) 399 [arXiv:0904.3830 [astro-ph.CO]].

4. M. Cirelli, F. Iocco and P. Panci, JCAP 0910 (2009) 009 [arXiv:0907.0719 [astro-ph.CO]].

5. M. Cirelli, P. Panci and P. D. Serpico, Nucl. Phys. B 840 (2010) 284 [arXiv:0912.0663 [astro-ph.CO]].

6. M. Pospelov and A. Ritz, Phys. Lett. B 671 (2009) 391 [arXiv:0810.1502 [hep-ph]].

7. N. Arkani-Hamed, D. P. Finkbeiner, T. R. Slatyer and N. Weiner, Phys. Rev. D 79 (2009) 015014 [arXiv:0810.0713 [hep-ph]].

8. A. Ibarra and D. Tran, JCAP 0902 (2009) 021 [arXiv:0811.1555 [hep-ph]].

9. E. Nardi, F. Sannino and A. Strumia, JCAP 0901 (2009) 043 [arXiv:0811.4153 [hep-ph]]. 